\title{
La Conferencia de Yalta y la enfermedad cerebrovascular
}

\section{The Yalta Conference and cerebrovascular disease}

\author{
Amado Jiménez-Ruiz ${ }^{*}$ y Adriana Ruiz-Razura²

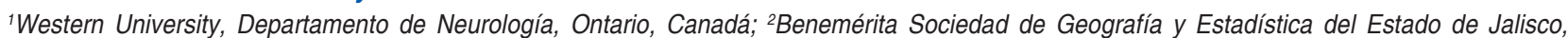 \\ Centro Universitario de Arte, Arquitectura y Diseño, Jalisco, México
}

Una persona inteligente resuelve un problema. Una persona sabia lo evita. Albert EINSTEIN

Leímos con atención el artículo titulado "Cáncer de cabeza y cuello. Su impacto en la historia de la humanidad", de José Francisco Gallegos Hernández, publicado en el número 2 de 2020 de Gaceta Médica de México. ${ }^{1}$ Esa reseña histórica deja el mensaje de que el cáncer puede ser curable en etapa temprana y que la prevención constituye el mejor tratamiento. Creemos que esto es cierto para la mayoría de las enfermedades y a manera de muestra, una fotografía (Figura 1).

En 1945, la Conferencia de Yalta marcó el final de la Segunda Guerra Mundial. Una famosa fotografía durante la reunión muestra a los líderes victoriosos de esa gran conflagración. Estados Unidos, el Reino Unido y la Unión Soviética discutían sobre la reorganización de Europa después del conflicto bélico. A la izquierda, Winston Churchill, seguido de Franklin D. Roosevelt y, al final, Joseph Stalin; los tres hombres más poderosos del planeta en ese momento. Sin embargo, existían otras similitudes: los tres murieron por complicaciones cerebrovasculares, incluidas isquemia y hemorragia cerebral; ;-4 los tres eran fumadores (en la fotografía puede observarse que uno sostiene un puro y el otro, un cigarrillo), hipertensos y obesos; los tres representan el arquetipo del paciente con una enfermedad que podría haberse prevenido con adecuado control de los factores de riesgo. Seguramente si estos personajes hubieran sobrevivido a sus afecciones vasculares, la historia

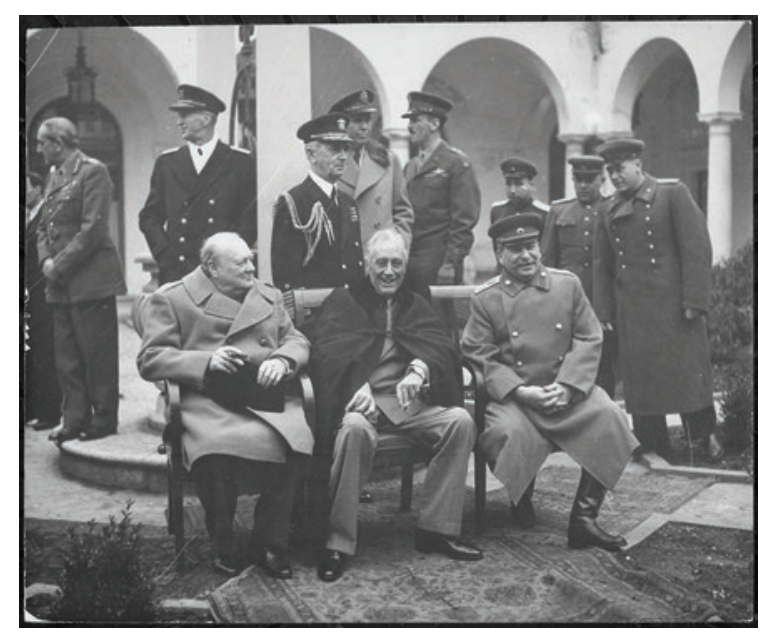

Figura 1. Conferencia de Yalta (fuente: imagen del dominio público).

hubiera sido otra. Coincidimos con Gallegos Hernández en que la prevención sigue siendo un pilar fundamental de la ciencia médica y debemos considerarla una prioridad siempre.

\section{Bibliografía}

1. Gallegos-Hernández JF. Cáncer de cabeza y cuello. Su impacto en la historia de la humanidad. Gac Med Mex. 2020;156:104-109.

2. Jones JM, Jones JL. Presidential stroke: United States presidents and cerebrovascular disease. CNS Spectr. 2006;11:674-678.

3. Ali R, Connolly ID, Li A, Choudhri OA, Pendharkar AV, Steinberg GK. The strokes that killed Churchill, Roosevelt, and Stalin. Neurosurg Focus. 2016;41:E7.

4. Barth RF, Brodsky SV, Ruzic M. What did Joseph Stalin really die of? A reappraisal of his illness, death, and autopsy findings. Cardiovasc Pathol Off J Soc Cardiovasc Pathol. 2019;40:55-58. 